\title{
Production Application Performance Data Streaming for System Monitoring
}

\author{
RAMIN IZADPANAH, University of Central Florida \\ BENJAMIN A. ALLAN, Sandia National Laboratories \\ DAMIAN DECHEV, University of Central Florida \\ JIM BRANDT, Sandia National Laboratories
}

In this article, we present an approach to streaming collection of application performance data. Practical application performance tuning and troubleshooting in production high-performance computing (HPC) environments requires an understanding of how applications interact with the platform, including (but not limited to) parallel programming libraries such as Message Passing Interface (MPI). Several profiling and tracing tools exist that collect heavy runtime data traces either in memory (released only at application exit) or on a file system (imposing an I/O load that may interfere with the performance being measured). Although these approaches are beneficial in development stages and post-run analysis, a systemwide and low-overhead method is required to monitor deployed applications continuously. This method must be able to collect information at both the application and system levels to yield a complete performance picture.

In our approach, an application profiler collects application event counters. A sampler uses an efficient inter-process communication method to periodically extract the application counters and stream them into an infrastructure for performance data collection. We implement a tool-set based on our approach and integrate it with the Lightweight Distributed Metric Service (LDMS) system, a monitoring system used on large-scale computational platforms. LDMS provides the infrastructure to create and gather streams of performance data in a low overhead manner. We demonstrate our approach using applications implemented with MPI, as it is one of the most common standards for the development of large-scale scientific applications.

We utilize our tool-set to study the impact of our approach on an open source HPC application, Nalu. Our tool-set enables us to efficiently identify patterns in the behavior of the application without source-level knowledge. We leverage LDMS to collect system-level performance data and explore the correlation between the system and application events. Also, we demonstrate how our tool-set can help detect anomalies with a low latency. We run tests on two different architectures: a system enabled with Intel Xeon Phi and another system equipped with Intel Xeon processor. Our overhead study shows our method imposes at most $0.5 \%$ CPU usage overhead on the application in realistic deployment scenarios.

\footnotetext{
This work is supported by Sandia National Laboratories. Sandia National Laboratories is a multimission laboratory managed and operated by National Technology and Engineering Solutions of Sandia LLC, a wholly owned subsidiary of Honeywell International Inc., for the U.S. Department of Energy's National Nuclear Security Administration under contract DE-NA0003525. This paper describes objective technical results and analysis. Any subjective views or opinions that might be expressed in the paper do not necessarily represent the views of the U.S. Department of Energy or the United States Government.

Authors' addresses: R. Izadpanah and D. Dechev, University of Central Florida, Department of Computer Science, 211 Harris Center (Building 116), 4000 Central Florida Boulevard, Orlando, FL 32816; emails: \{izadpana, dechev\}@cs.ucf.edu; B. A. Allan and J. Brandt, Sandia National Laboratories, PO Box 5800, MS 0823, Albuquerque, NM 87185; emails: \{baallan, brandt\}@sandia.gov.

ACM acknowledges that this contribution was authored or co-authored by an employee, contractor, or affiliate of the United States government. As such, the United States government retains a nonexclusive, royalty-free right to publish or reproduce this article, or to allow others to do so, for government purposes only.

(C) 2019 Association for Computing Machinery.

2376-3639/2019/04-ART8 \$15.00

https://doi.org/10.1145/3319498
}

ACM Trans. Model. Perform. Eval. Comput. Syst., Vol. 4, No. 2, Article 8. Publication date: April 2019. 
CCS Concepts: • General and reference $\rightarrow$ Measurement; Performance; Evaluation; Experimentation; Empirical studies; Metrics; • Computing methodologies $\rightarrow$ Massively parallel and high-performance simulations; Simulation evaluation; Modeling methodologies; • Software and its engineering $\rightarrow$ Software performance;

Additional Key Words and Phrases: Application and system monitoring, performance data streaming, application profiling

\section{ACM Reference format:}

Ramin Izadpanah, Benjamin A. Allan, Damian Dechev, and Jim Brandt. 2019. Production Application Performance Data Streaming for System Monitoring. ACM Trans. Model. Perform. Eval. Comput. Syst. 4, 2, Article 8 (April 2019), 25 pages.

https://doi.org/10.1145/3319498

\section{INTRODUCTION}

Making high-performance computing (HPC) applications efficient and reliable at high process counts is challenging and requires a comprehensive knowledge of the application behavior and system state. Continuous system monitoring is necessary to gain this knowledge. HPC-monitoring tools collect global system information on resource utilization and system states such as network and storage usage, CPU and memory utilization, and environmental conditions. Also, events from the inside of applications can have critical performance implications [25, 34]. In this article, we present an approach to continuously collect application level performance data and expose them to HPC-monitoring tools.

Extracting application events, especially in a production environment, involves challenges such as minimizing the interference with computations, dealing with the tradeoff between the data accuracy and application efficiency and determining the appropriate time and location of data exposure. Application profiling tools typically take approaches based on large trace collection or statistical sampling of the program counter and call stack. Capturing application behavior with full details introduces overhead in memory, network bandwidth, and storage. Furthermore, this approach negatively impacts the application's ability to execute its operations and perform computations. However, it is challenging to choose a subset of events and compile proper statistical samples that represent the application's behavior of interest with reasonable accuracy and efficiency. Many of the performance-monitoring libraries accumulate data and release them after the program termination [44, 45, 47]. Revealing the performance data at the end of run could be helpful in some cases such as application tuning and optimization that is performed during development and before deployment. However, continuous system monitoring in production environments demands the collection and exposure of data during the runtime efficiently.

In this work, we present an approach to collect application level events and provide a production-time status of the application. Our approach can integrate with HPC-monitoring tools to support continuous system monitoring in production environments. Independent components of this approach make it suitable to work with different programming paradigms and monitoring tools. In our approach, we utilize an efficient inter-process communication (IPC) method to convey data from the application profiler, which is the data provider, to the data consumer in the performance-monitoring tool. The application profiler collects data through dedicated counters that are embedded in the application's points of interest. The HPC-monitoring tool exposes the provided data at periodic intervals during the application execution. The data exposure at the execution time enables efficient software-level performance data streaming, which is necessary to provide monitoring services in production systems seamlessly. 
Runtime performance analysis tools employ event tracing through instrumentation or sampling approaches to monitor and evaluate HPC applications [1, 31, 32, 37, 44, 45, 47]. Methods used for instrumentation include modifying the source code, changing the binary code, and function call interception through linking with specialized libraries such as tools based on Message Passing Interface (MPI) profiling layer [29]. An extensive amount of information about the desired parts of the application with details is collected to characterize its behavior. However, these methods usually add significant overhead to the system regarding memory, network bandwidth, and storage. The high overhead of instrumentation makes these methods unsuitable to apply to many code regions in applications or in production. Sampling methods collect statistical performance data at intervals or when interrupted by external events [44]. In this approach, performance analysts should tune the sampling intervals to achieve the optimal solution based on specific efficiency goals. Profiling tools that work based on this model provide the most accurate data at high frequencies with a considerably high cost. However, they are more efficient at low frequencies but less informative and prone to detail loss [3]. Current approaches either lack the necessary efficiency to be utilized in production systems or support only post-mortem analysis that does not present online data about application events during the execution. Also, developers face challenges when analyzing applications that scale to larger parallel systems [9].

The novelty of our approach is its efficiency in providing exposure of software-level events while the application is running. In our approach, we collect events through software-level counters and expose them to the data consumer in the HPC-monitoring tool during the application execution. We design and implement a tool-set based on our approach and demonstrate its impact using a case study of the analysis of a scientific application. Our tool-set consists of three components: application profiler, shared memory index, and a sampler. The application profiler collects information about the software-level events. The shared memory index provides a mechanism to locate the data collected by the application profiler. The sampler utilizes the shared memory index to expose the data collected by the application profiler instances periodically.

We integrate our approach with the Lightweight Distributed Metric Service (LDMS) system [2], a monitoring system used on large-scale computational platforms [9]. LDMS provides the infrastructure to gather streams of performance data efficiently while keeping the overhead low. The scalability of LDMS allows us to build a tool-set to monitor large-scale applications. Furthermore, the design of LDMS supports plug-in sub-modules [17]. The sampler component of our tool-set operates as an LDMS sampler plug-in and is independent of the specific metrics collected or the application. We demonstrate our approach using applications implemented with MPI. MPI is one of the most common standards for the development of large-scale scientific applications. The MPI profiling interface (PMPI) [29] allows us to instrument many HPC applications without the modification of their source or binary code. The application profiler component of our tool-set is implemented to profile use of the MPI API, though our method may be adapted to any other instrumentation approach producing counters.

Our streaming-based method enables runtime operational analysis and troubleshooting in HPC applications. By continuous, efficient, and direct access to application events, monitoring tools can generate software performance data stream as well as the general system state. The software-level metrics exposure supports the performance analysis based on exploring correlations between the system events and application internal events. This analysis helps users to understand the application behavior and possible performance bottlenecks. Our approach is not trace based, and it avoids generating large datasets in the application's hardware environment. It allows processing performance data elsewhere while the application is running. Data stream processing allows for gaining immediately useful insights by computing functional combinations of data. Our tool-set can benefit a researcher doing application characterization without interfering with the 
computation. For example, our tool-set can provide insights that can be used in combination with other data sources in an automatic application phase detection approach. This can be achieved without looking at source code or binary instrumentation.

We demonstrate the impact of our method using an open source HPC application, Nalu [16]. Nalu has been chosen as one of the representative simulation codes to be used as a performance benchmark for the Trinity Capability Improvement Metric [4]. It is a representative of implicit codes that have been developed under the Advanced Simulation and Computing (ASC) [5] program. We show how our tool-set enables us to efficiently identify patterns in the behavior of the application without any scientific domain knowledge or access to the source code. We leverage LDMS to collect system-level performance data as well as software-level data and explore the correlation between the system and application events. Also, we show how our tool-set enables quick issue mitigation by assisting in the detection of anomalies in the behavior of the application. We run tests on two different architectures to understand the interaction of our tool-set with the operating system and applications on different HPC platforms. These architectures include a system enabled with the latest generations of Intel Xeon Phi code-named Knights Landing and another system equipped with Intel Xeon processor.

Our overhead study shows our method imposes at most $0.5 \%$ CPU overhead on the application.

This article makes the following contributions:

- We present the first approach to stream performance data from software-level events into an HPC system-monitoring infrastructure. This approach provides external access to the application profiling data during execution in a tunable manner with low overhead in the production environment.

- We implement a tool-set based on our approach and integrate it with LDMS to demonstrate its impact. Application performance data streaming enables runtime analysis and feedback to both the application and the system.

- We present a case study demonstrating a large-scale scientific application characterization using our approach without relying on access to application output files.

- We present a case study demonstrating how our approach can help detect anomalies in an application's behavior.

The rest of this article proceeds as follows. We discuss the related work in Section 2. In Section 3, we present the background on the system-monitoring tool. In Section 4, we explain our approach and design and implementation details of our tool-set. We present the experimental evaluation and discuss the results in Section 5. Finally, we conclude in Section 6.

\section{RELATED WORK}

A wide range of performance-monitoring and -analysis tools exist that rely on tracing or profiling approaches to collect data.

Cloud-based tools such as Graphite [14] and Prometheus [39] provide a different set of services for applications and systems that are hosted on the cloud. Graphite relies on third-party tools for data acquisition services and is mainly used for storage and visualization purposes [28, 48] in conjunction with monitoring data collection tools in the cloud environment. While Graphite and its data collectors provide a convenient monitoring mechanism by supporting string format communication and script-based commands, these characteristics limit the frequency, accuracy, and latency of data collection. Prometheus text-based exposition format does not separate metadata from data, limiting the sampling frequency and latency due to the parsing. These limits in addition to the overhead on the bandwidth and computing resources availability to the primary applications prevent wide applicability of these tools in HPC environments. While these tools require 
extremely capable nodes for data aggregation, our tool builds upon LDMS, which can aggregate on the same class of hardware the collectors run on. In addition, unlike the equipment requirements of cloud-based solutions like these tools, we build our tool upon LDMS that has demonstrated that it requires only two aggregator nodes to serve more than 27,000 clients on NCSA Blue Waters [2, $38]$ and a similar number of nodes on Trinity [12, 13, 33].

Some general-purpose system-monitoring tools mainly focus on monitoring and analyzing resource utilization in the system and not the application performance. Some tools such as Ganglia [36] have limitations in scalability, and Nagios [40] has a different purpose of failure alerting. LIKWID Monitoring Stack [41], which targets small-to medium-sized commodity clusters, focuses on utilizing hardware performance counter data and does not provide software-level performance metrics. GUIDE [46] is another scalable tool for data collection and analysis that focuses on the entire HPC ecosystem and does not provide application-level insights. Our tool-set streams software-level performance data and has a broader purpose than these tools.

The gprof tool [20] supports both sampling and instrumentation mechanisms separately for sequential programs. It creates a report at the end of execution and is not considered a scalable tool for monitoring large-scale parallel applications. HPCToolkit [1] provides a performance analysis framework that directly analyzes the binaries of applications without instrumentation. From this perspective, HPCToolkit and other tools based on this model [18, 24, 43] are mostly used for MPI call-path profiling and performance tuning during development, releasing the performance data after program termination. We demonstrate our approach using MPI applications. However, our design is not limited to a specific programming pattern. Our tool-set combines sampling and profiling to stream accurate data efficiently and supports runtime analysis.

Periscope [7] takes a distributed search approach to detect performance issues specified by users and relies on other libraries to collect the performance data. The iterative strategy of Periscope requires the application to follow a specific programming pattern with a region, such as the main loop in scientific simulations, that repeats during the execution. TAU [37] offers offline performance analysis of applications based on tracing and profiling approaches and generates the result after the application termination. Vampir [31] provides visualization of performance traces and profiles generated by other tools. Scalasca [19] performs post-run analysis using parallel trace replay for specific performance issues.

TAU could be used as a partner tool in the instrumentation of modifiable applications. However, the binary or source rewriting mechanisms used by tools like TAU limit their applicability. These limitations make the tools not suitable choices for many production HPC settings where application builds have been through specific verification and validation steps. Our streaming-based approach enables runtime analysis and does not enforce a specific programming paradigm.

Periscope, TAU, Vampir, and Scalasca use Score-P [32] measurement system as the underlying layer to record performance data. Score-P instruments applications and stores the performance information in forms of profiles or traces. Besides the support for the offline analysis, Score-P provides the interface to Periscope to perform the online analysis. However, it does not generate a stream of performance data during the execution of the application. Tools that use Score-P must follow a specific work-flow, which typically involves recompiling and relinking the application using the Score-P compiler. Our implemented tool-set does not require the user to recompile or relink their application for monitoring.

MPItrace [44], IPM [45], and mpiP [47] specifically support the performance analysis for MPI applications. MPItrace supports both tracing and sampling-based approaches. The sampling mechanism is built on top of Performance API (PAPI) [10] and supports sampling based on available hardware counters. IPM and mpiP collect detailed and statistical performance data, respectively, and both generate the results after the program termination. 
We implement a tool-set based on our approach and demonstrate it using MPI applications. Our tool-set enables operational runtime analysis using performance data streaming. By integrating this approach with LDMS, we can explore the correlations between events that are happening in different layers of the software and hardware stack.

\section{LDMS MONITORING FRAMEWORK BACKGROUND}

Our approach leverages LDMS's capabilities in data transport and aggregation that enables continuous monitoring in large-scale systems. In the LDMS framework, daemons employ plug-ins on the monitored resources for data sampling. In a typical data collection process, daemons on compute nodes, which are configured as sampler daemons, keep the collected data in a structure called metric sets in the memory. Daemons update the metric set with the new metric values using sampler plug-ins with a configured frequency. Daemons can also play the role of an aggregator.

Aggregator daemons can aggregate data from other LDMS daemons over various transports for arbitrary purposes such as feeding data to other consumers, performing low-latency analysis and writing out to some permanent storage system $[2,26]$. Aggregators shift the load and overhead of storage and aggregation to cluster service nodes that do not run HPC applications. This offloading reduces the overhead on compute node as much as possible and eliminates possible interference between computations and storage.

LDMS has been used in monitoring large-scale HPC systems such as NCSA's Blue Waters [38] Cray XE/XK system with 27,648 nodes. LDMS has performed efficiently on large-scale production systems, and overhead assessments have demonstrated no significant detrimental system impact $[2,12,13,38]$. LDMS enables low-overhead performance data streaming using the pull model of aggregators that periodically fetch data from the samplers. This pulling mechanism utilizes the Remote Distributed Memory Access protocol to unburden the compute nodes of the required functionality and overhead for sending data, storage, and failover handling. Clients can connect to aggregators and consume the data stream for various purposes such as analysis and storage.

\section{METHODOLOGY}

In this section, we describe our approach to collect and expose performance data. We start with an overview of our model and the system components. We introduce each component and explain the design decisions. Next, we explain the work-flow and how different components work together to stream performance data. Finally, we discuss the challenges that we face in our tool-set's design.

\subsection{System Overview}

Figure 1 displays a high-level overview of the system. Our design consists of three components: an application profiler, a shared memory index, and a sampler. The application profiler, which is described in Section 4.2, collects information about the software-level events. The shared memory index, which is described in Section 4.3, provides a mechanism to access the data collected by the application profiler. The sampler, which is described in Section 4.4, utilizes the shared memory index to expose the data collected by the application profiler periodically.

The shared memory index is identified using a name that is assigned to a region in the shared memory area on the system. The sampler and the application profiler are configured with the same index name to access the shared memory index. The shared memory index consists of entries that each correspond to an application process being monitored. The application profiler instance puts data collected about its events in a specific location in the shared memory. Each index entry contains information about this shared memory location. The sampler stores the information about each application in an instance of a data type called box. A box holds information such as the metric set description and the data collected by the application profiler. 


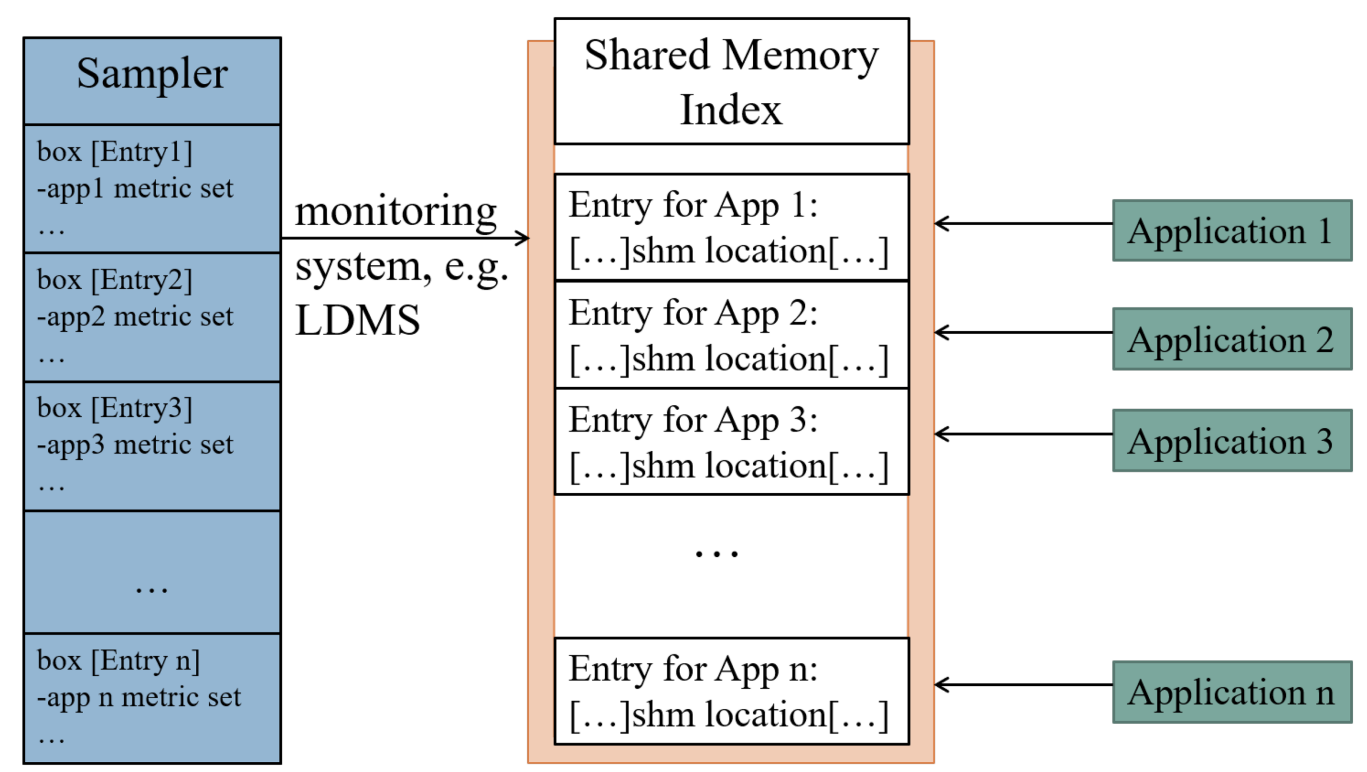

Fig. 1. A high-level overview of the system components. Application profilers collect performance data and share them with the sampler using the shared memory index. Shared memory index consists of one entry per application. Sampler allocates a box for each entry in the index.

As we explain in Section 4.5, these components cooperate with each other in our tool-set to stream performance data that are collected from the application. In Section 4.6, we discuss the challenges involved in our design.

\subsection{Application Profiler}

In our approach, the application profiler is the data provider to the sampler. The profiler collects information about application-level events. The application profiler assigns dedicated counters to each application event defined at the software level to perform the data collection. The profiler provides information about events to the sampler via the shared memory index. This flexible design enables us to develop customized profilers to accommodate any specific type of application and programming pattern. The programming paradigm used and the application developer determine the types of events to be collected and monitored.

We design and develop an MPI profiler as a proof of concept. Our MPI profiler leverages PMPI to collect data from the application. This method requires no modifications of either MPI applications or libraries. We rely on the Linux library preloading feature to inject our profiler at runtime. This mechanism imposes no burden on the programmer to recompile the application, except in statically linked applications.

Our MPI profiler provides a query interface to retain flexibility and convenience to study various events with different properties. In this interface, the user can specify events with particular characteristics and features that need to be measured. This mechanism enables the user to focus on the essential features that have impacts on a specific aspect of application behavior rather than being exposed to a massive amount of data that can be generated. Multiple aspects may be specified as shown by an example pseudo-code in Listing 1 . This query instructs the MPI profiler to perform the measurement per MPI rank and only measure the message size and number of calls for message-based events where the message handled by that event is greater than 10 bytes. 


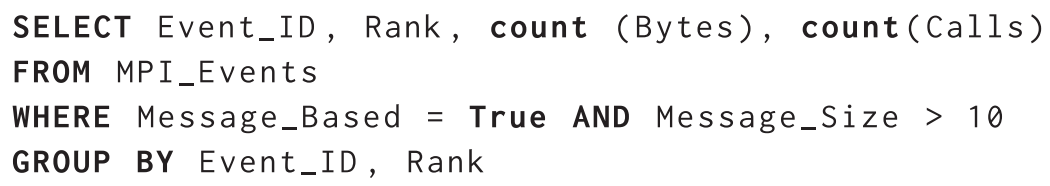

Listing 1. Example pseudo-code that demonstrates the functionality of MPI profiler query interface.

The application profiler uses the shared memory index to expose events to the sampler. In Section 4.3, we explain the shared memory index design.

\subsection{Shared Memory Index}

To access the information about software-level events in applications, we need to employ a method of IPC. Various communication facilities allow processes to exchange data with one another. These facilities can be divided into two categories: Data-transfer and Shared memory [30]. The first type of communication involves kernel functions in the data transfer, but in shared memory approaches, processes can communicate with each other by placing data in a shared memory region. This direct communication makes the shared memory approach a fast method, because it requires few system calls or kernel operations. Unlike data-transfer facilities, shared memory allows one process to make the data visible to any number of processes that share the same memory region. The shared memory approach enables a process to access shared data like any other memory area in its virtual address space. This model fits well with application designs that need to maintain a shared state between multiple processes, such as the approach we present in this article.

Most UNIX-based systems provide the support for shared memory. This type of IPC should be employed cautiously as the operations on the shared memory may need to be synchronized. The synchronization could negatively impact the advantages of fast communication in shared memory approaches depending on the use case. In our implementation, there are a few scenarios, e.g., initial configurations, that we use synchronization facilities such as semaphores to protect shared resources.

We choose POSIX shared memory over System V shared memory in our design as POSIX features better fit our implementation needs. The advantages of POSIX IPC include the simplicity of the interface, consistency with the traditional UNIX file mode, and support for reference counted objects [30]. Furthermore, POSIX IPC mechanisms are guaranteed to be thread-safe, but System V IPC techniques do not provide such a guarantee [42]. In our approach, multiple processes, including parallel applications, utilize shared resources. In Section 4.6, we discuss how we use reference counts to manage shared resources.

In our implementation, the shared memory index consists of entries corresponding to application processes being monitored. Each entry stores information about the application instance including the location in the shared memory of the application instance's profiling data. This information is shared with the sampler using the assigned index entry for the application. The data layout that is shared between the application profiler and sampler is determined dynamically at runtime. This data layout is called a metric set, which defines a collection of metrics and provides data about it.

Figure 2 displays the general structure of a metric set that is stored in the specified shared memory region within our tool-set. There are three chunks of contiguous memory associated with each metric set. First is the metadata that provides general information about the metric set as a whole. The number of monitored events is an example of such information that is stored in the metadata. Second is the general information describing the elements of the data chunk, such as the event name. The final piece of data represents the counter values correspond to collected events by the application profiler. No history is retained within a profiler or the shared memory index, and the allocated memory is overwritten as the profiler provides new information about an event. 


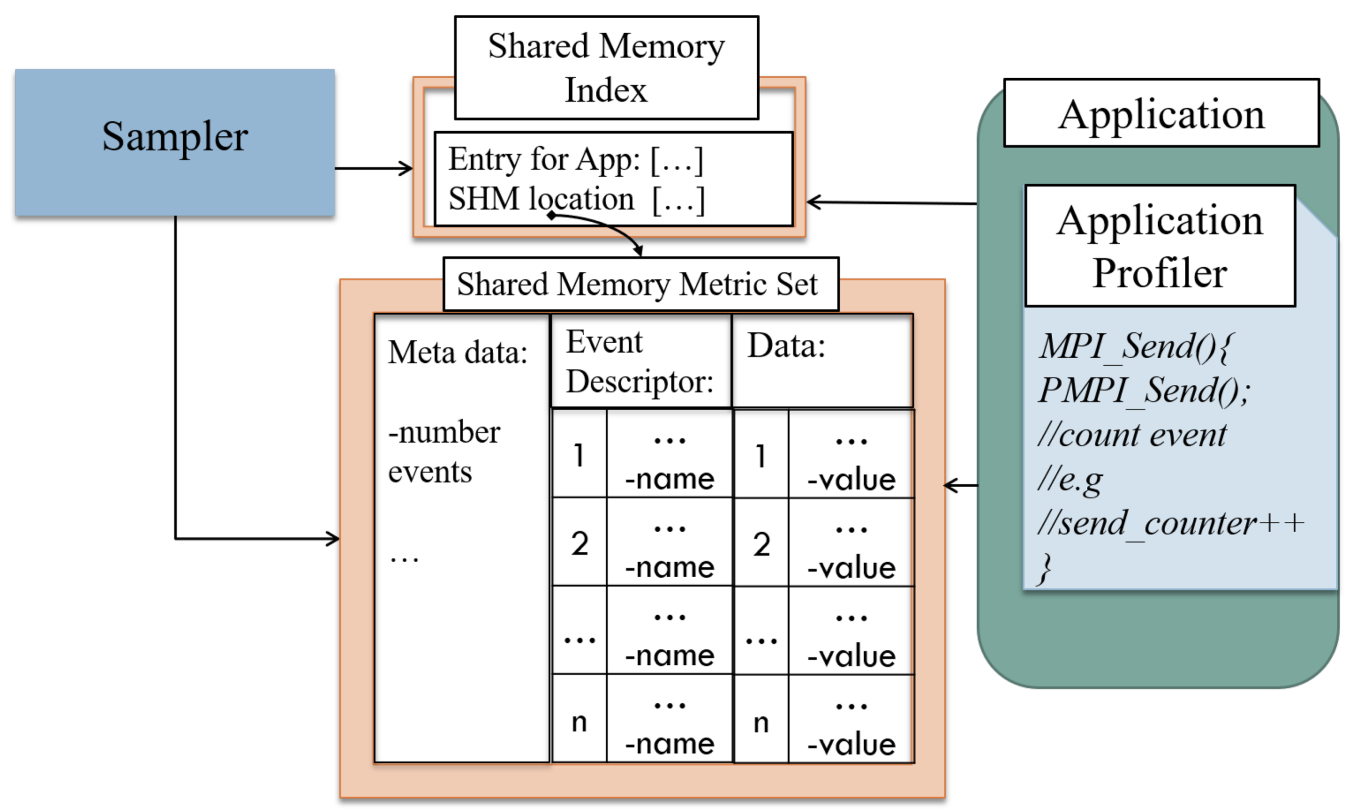

Fig. 2. SHM metrics set.

The shared memory index allows the application profiler and sampler to access the events information simultaneously. In Section 4.4, we describe the role of sampler within our tool-set.

\subsection{Sampler}

We leverage the LDMS for data collection, transport, and aggregation. The typical process of data collection starts with running LDMS daemons on compute nodes. These daemons, which are configured as sampler daemons, store the collected data in LDMS metric sets. A sampler daemon stores only the latest set of values for each of its metric sets, and no sample history is retained within a plug-in or the host daemon.

The streaming part of our methodology relies on the sampler component. The sampler is a daemon plug-in that periodically copies data provided by a source on a monitored computing node and exposes the collected data to consumers. The sampling frequency, which is defined by the user, determines the resolution of data. Running a sampler that utilizes the application profiler's shared memory as the data source allows us to stream application performance data related to softwarelevel events while the program is running.

We implement the plug-in shm sampler within the LDMS performance-monitoring framework. This sampler stores the information from each application profiler instance in an instance of a data type called box. A box holds information such as the metric set collected by the application profiler and other information that is required by LDMS infrastructure. The sampler dynamically discovers the metric set defined by the profiler and creates the box corresponding to it.

In Section 4.5, we explain how different components within our tool-set cooperate to generate the stream of application-level performance data.

\subsection{Work-flow}

Figure 3 displays the typical work-flow in our approach, annotated with the associated actions for each component. Both sampler and application profiler should agree on an index name that is used to obtain the access to the shared memory index. This index is created at the first call to open 


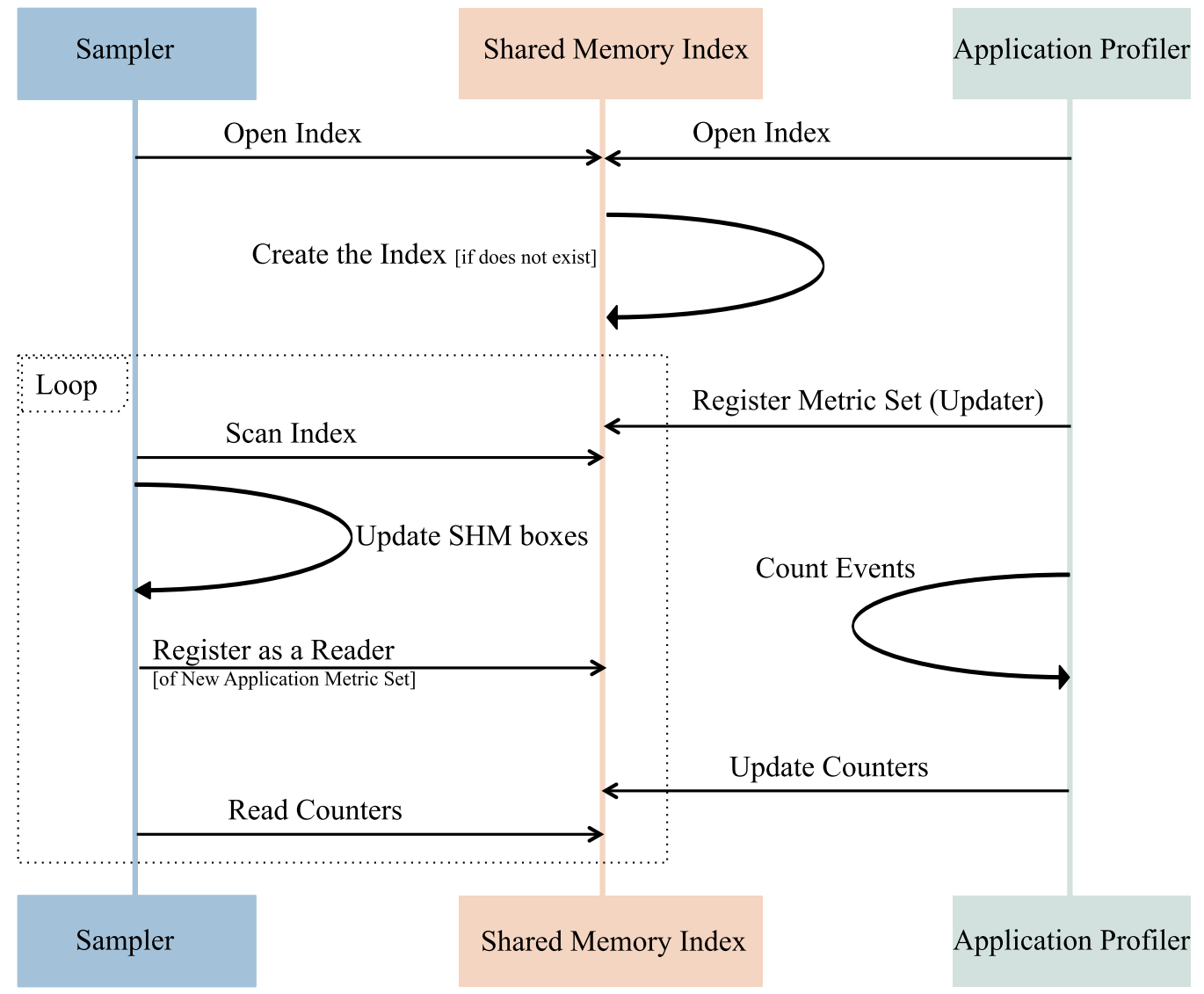

Fig. 3. The work-flow of our methodology.

index by any process. Unix shm_open API provides a file descriptor that can be used to handle the shared memory region with the specified name. Subsequent calls to open index do not allocate a new area in the shared memory, and the file descriptor will be used to access the index. Each process creates a memory mapping in its virtual address space using the provided file descriptor. All processes can access the same index and use the mapped memory to share information.

The user configures the application profiler to monitor events of interest. After the configuration, the profiler provides the required information about events to the shared memory index. At this step, the application profiler registers itself as the updater of a metric set in the index using the provided information. The shared memory index allocates an entry to the application and creates a shared memory metric set as specified in Figure 2.

The sampler periodically scans the index to find new or modified entries and updates its local boxes. The sampler detects changes in the metric set using a generation number associated with each index entry allocated to an application. When an application profiler modifies the configurations of events, the generation number of that entry is incremented. The sampler, as a data consumer, determines if the generation number it has stored locally matches that associated with the current produced data by the profiler.

For each new application, the sampler registers itself as a reader of its metric set. Also, the sampler configures a new metric set in the performance-monitoring library infrastructure. After the setup, the application profiler counts the specified events, as they happen in the application, 


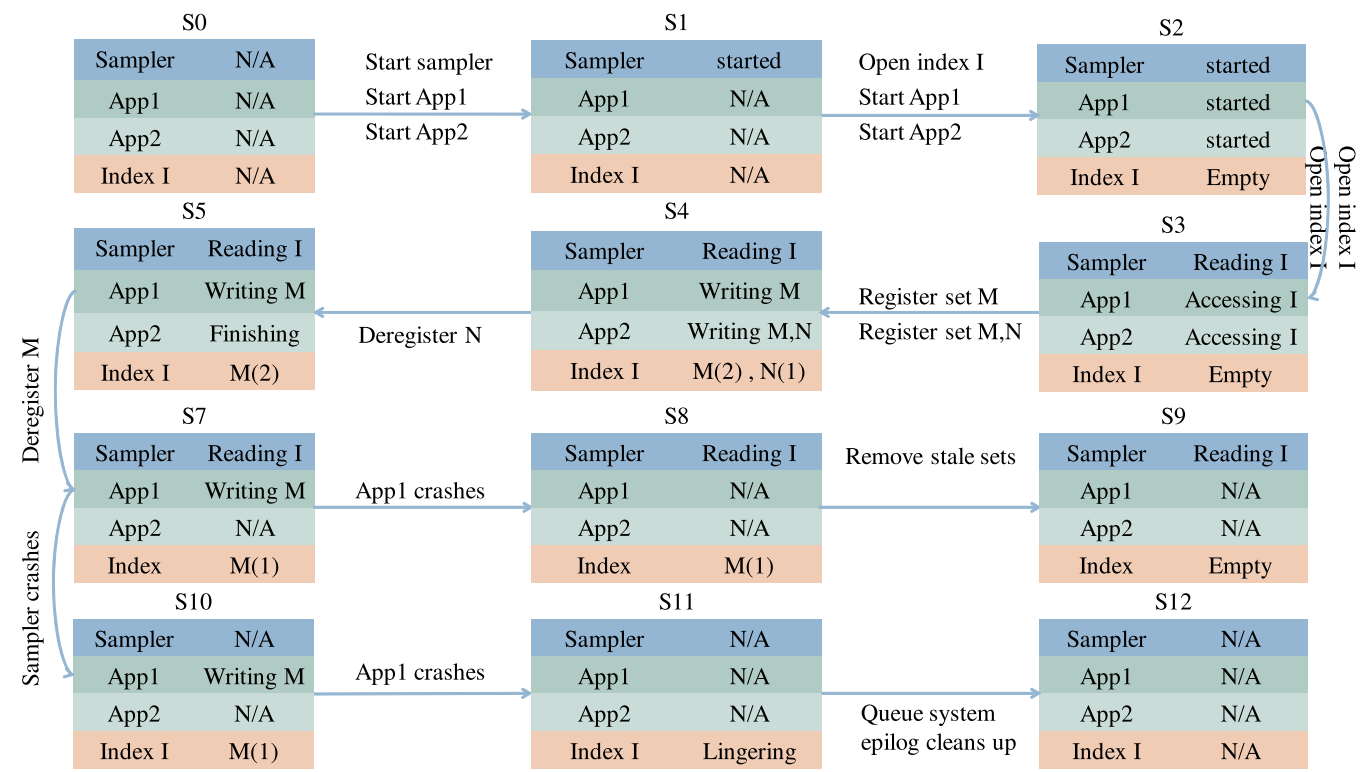

Fig. 4. Index management. The second column of each table represents the state for the sampler, applications and the index. Transitions between states are displayed using arrows, which are annotated with the associated action.

and updates the counters associated with events. The sampler reads the counter values at intervals and exposes them to the consumers.

This work-flow within our tool-set yields application performance data streaming for system monitoring. This cooperation between multiple processes in a shared environment is not without challenges. In Section 4.6, we explain some challenges involved in this work-flow and how we deal with them.

\subsection{Handling Process Failures}

In our approach, the index is shared between the sampler and the application processes (e.g., MPI ranks). The shared resources used in the index and its entries need to be handled carefully in different scenarios such as crashes. Figure 4 exhibits different scenarios using the sampler and two applications. We explain how we deal with shared resources in different situations. In this figure, we have 12 different sets of states and each set shows the state of the sampler, two sample applications called app 1 and app 2, and an index named I.

We start from the initial state where applications and the sampler are not available. We assume that the index name is somehow defined in this environment (e.g., defined by the user). Next, we start the sampler and the applications, and we assume that the sampler wins the race and starts first. Since the sampler starts first and finds out that the index is not available, it creates index I.

Now, we are in state 2 that the sampler and applications are started, and the index is created. Applications open the index and register their metric sets in the index. We assign reference counts to metric sets defined in index entries to keep track of each indexed set. In our example, state 4 displays that the metric set $\mathrm{M}$ with two references and the metric set $\mathrm{N}$ with one reference is defined in the index. App 1 is writing into the metric set $M$ and app 2 is writing into the metric sets $\mathrm{M}$ and $\mathrm{N}$. The sampler is reading all sets included in index I.

Now, let us assume that app 2 finishes its execution. App 2 deregisters metric sets $\mathrm{M}$ and $\mathrm{N}$ before it terminates. We decrement the reference count for any set that is deregistered. For any set 
that the reference count value is zero, the shared resources can be cleaned. If the reference count value for a set is not zero, then it means that another application is writing into this set. In our example, state 7 shows that app 2 is no longer running and app 1 is still writing into set $\mathrm{M}$. Index I contains a metric set $\mathrm{M}$ with one reference, but metric set $\mathrm{N}$ does not exist anymore, because its reference count dropped to zero and was cleared from the index.

Starting from state 7, we cover two crash scenarios. First, we assume that app 1 crashes in the middle of its run. This crash causes a transition to state 8. At this state, none of the applications are running. Index I still exists with the metric set $\mathrm{M}$ with one reference and the sampler is still reading from this index. To handle this type of crash we use a timeout concept. After a period of inactivity since the last update in the metric set by the application, the sampler may assume that the set is no longer in use. At this step, the sampler notifies the index to remove the stale set. We end up in state 9 where the sampler is running, and the index is empty.

Another possible crash scenario, starting from state 7, happens when the sampler quits unexpectedly in the middle of the execution. The unexpected quit results in a transition to state 10 , where index I exists with the metric set $M$ and app 1 is running and writing into set $M$. If app 1 finishes its execution safely, before terminating, then the application profiler cleans the shared resources. If the last writer upon its deregistration finds out that there are no active readers, then it can unlink the index file. The profiler figures this out using either read timeout or reference counts. However, if app 1 crashes, we will end up in state 11 where no program is running and index I still exists without any owner. At this step, the queue system epilog cleans up the idle index file between jobs.

Utilizing reference counts, and read and write timeout mechanisms enables us to manage the allocated resources in different use cases including failures in any of the involved processes.

\section{EXPERIMENTAL EVALUATION}

In this section, we demonstrate the impact of our method using an open source HPC application, Nalu. We start with a brief introduction of Nalu. Next, we present a case study of using our toolset for performance monitoring and analysis of Nalu. Finally, we evaluate the overhead impact of using our tool-set when Nalu is running.

\subsection{Nalu}

Nalu is a massively parallel computational fluid dynamic application built on top of the Sierra Toolkit and the Trilinos solver Tpetra stack. Nalu has been chosen as a representative simulation code and performance benchmark for the Trinity Capability Improvement Metric [4]. It is a representative of implicit codes that have been developed under ASC program [5].

The results presented in Sections 5.2.1 and 5.2.2 are extracted from the waleElemXflowMixFrac3.5m model, which is available in the Nalu repository. We run this model using eight MPI processes on a KNL system as specified in Table 1. In Section 5.2.3, we use the milestoneRun model to run Nalu with thirty MPI processes.

\subsection{Case Study}

In this section, we present the case study we use to demonstrate one intended use of our tool-set. First, we explain how our tool-set can provide insights to assist with the process of revealing application phases. Next, we demonstrate how software-level performance data provided by our tool-set contributes to a better understanding of the application behavior by correlating to hardware-level metrics. Third, we show how our tool-set can help detect issues in the application execution in a timely manner. Finally, we demonstrate how our tool-set enables HPC users to monitor multiple scientific applications. 
Table 1. System Specifications

\begin{tabular}{|c|c|c|c|c|c|}
\hline System & $\begin{array}{c}\text { Processor Model } \\
\text { Clock }\end{array}$ & $\begin{array}{c}\text { Cores (Physical) } \\
\text { Threads }\end{array}$ & Memory & OS & Compiler \\
\hline KNL & Intel XEON Phi & 68 & 16GB MCDRAM & CentOS 7.3 & Intel 17.0.1 \\
& $7250 @ 1.4 \mathrm{GHz}$ & 272 & 96GB DDR4 & & \\
\hline Xeon & $\begin{array}{c}\text { Intel Xeon E5-2683 } \\
\text { @ } 2.10 \mathrm{GHz}\end{array}$ & 32 & 128GB DDR4 & CentOS 7.1 & Intel 17.0.1 \\
& 32 & & & \\
\hline
\end{tabular}

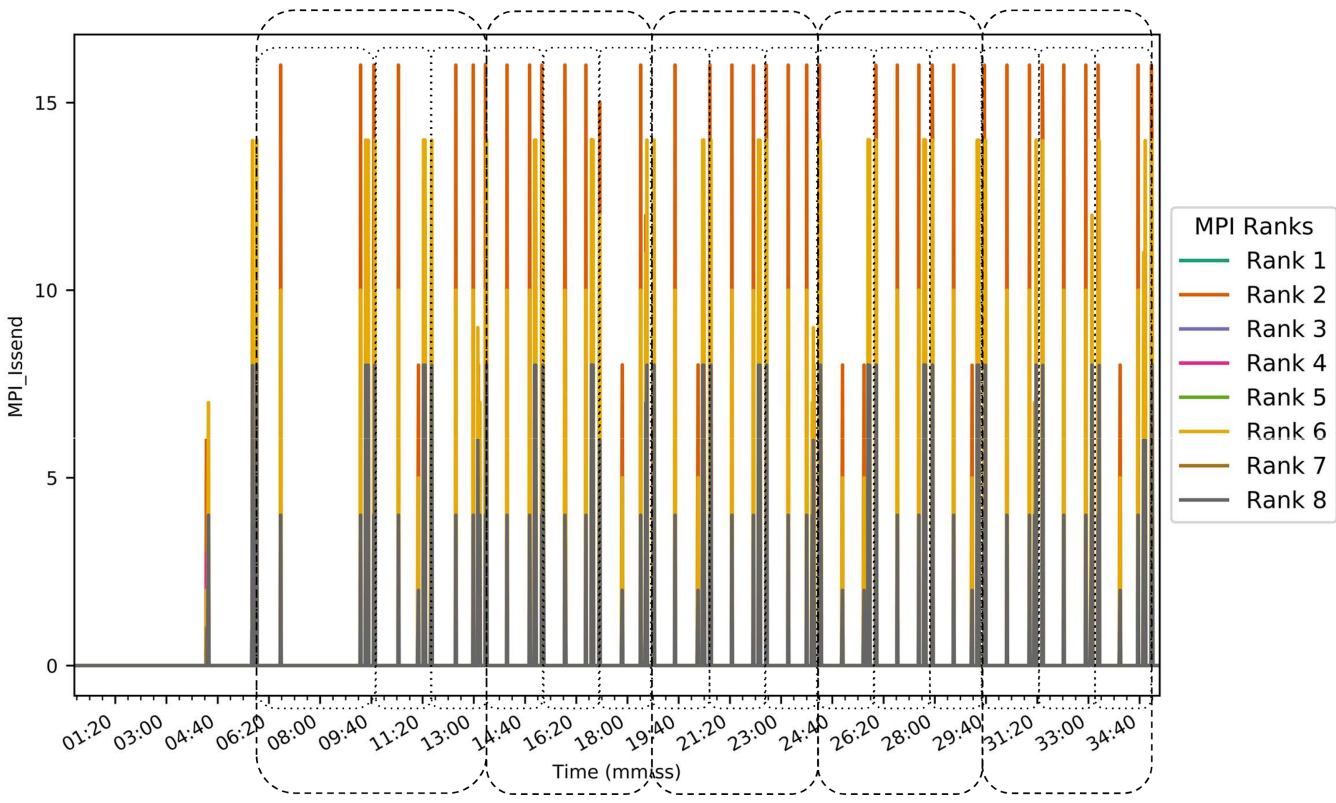

Fig. 5. Nalu phases.

5.2.1 Application Phases. Scientific applications typically execute in several phases. They usually start with an initialization phase and end with a final phase. In parallel scientific applications the intermediate computation phase typically consists of nested iterations. The computation phase usually dominates the application efficiencies and load balance. In this section, we show our toolset provides information that can be used as one data source in an automatic phase detection method.

Figure 5 exhibits the rate of changes in the number of calls to MPI_Issend function during the execution of Nalu on the waleElem model. Nalu starts with an initialization phase, which involves initial communications, initial value assignments to variables, and decomposition. We do not see any repetitive patterns during the initialization phase (which lasts for about 6 minutes). After finishing this initialization, the computation phase begins.

In Figure 5, regions surrounded by dashed bubbles demonstrate a periodic behavior in this application. We attribute this repetitive pattern to application iterations executed during the computation phase. To validate this, we add information about the application iterations to the log file produced by Nalu application. Figure 6 displays this information extracted from Nalu log file.

In both figures, we observe 6 minutes of initialization. Next, the computation phase begins, which consists of five timesteps as configured in Nalu input file. At each timestep, Nalu runs three 


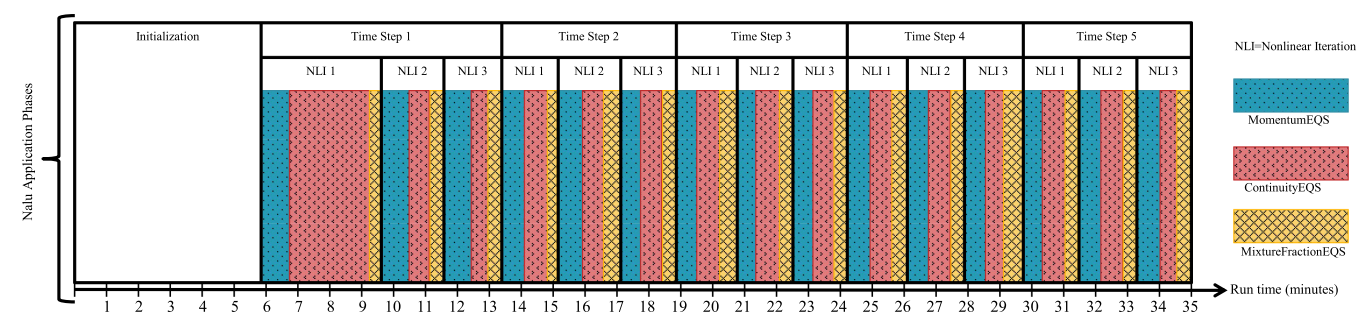

Fig. 6. Nalu phases extracted from log files.

nonlinear iterations. During each iteration, the application solves three equation systems. In Nalu application's structure, these equations are identified as MomentumEQS, ContinuityEQS, and MixtureFractionEQS. According to Nalu's manual, these systems are used by the WALE model to capture the asymptotic behavior for flows. By aligning Figures 5 and 6, we can derive application phases with the granularity of equation system iterations from the MPI data collected by our tool-set.

Exposing performance data in a streaming manner by our tool-set enables us to monitor the level of progress in the application as it is running. We do not need knowledge of the log file location or its contents to observe detailed application behavior and compare it to previous runs of the application. To facilitate the phase detection, our tool-set can be used as one data provider in combination with other data sources in an automatic application phase detection approach. This is an essential feature, particularly for system support staff lacking specific application knowledge. Our tool-set provides this information using collected MPI data with a negligible interference with computation. In Section 5.3, we study the overhead of using our approach.

\subsubsection{Correlations of Application-level Events with Other Performance Data Representing System} Events. Integrating our software performance counter collection approach with LDMS allows us to study how events happening at the hardware level are related to the application-level events. The LDMS framework provides several sampler plug-ins for the data collection from hardware performance counters. We can run several sampler plug-ins at the same time as our sampler. In this section, we use our tool-set to demonstrate a cursory exploration of the correlation between hardware and software metric time series.

In Figure 7, six graphs are plotted together. All graphs share the same $x$-axis that represents the runtime in minutes. The first graph from the top with "MPI_Issend" label on the $y$-axis displays the rate of calls per second to MPI_Issend function, which represents the application activities using software-level metrics. The second graph with "procnet_tx_bytes" label on the $y$-axis shows the byte rate of the data transmission by the network interface. The third graph with the $y$-axis labeled "meminfo_Dirty" represents the total amount of memory waiting to be written back to the disk. The fourth graph with "nfs_numcalls" label on the $y$-axis displays the rate of total RPC calls per second to NFS. The fifth graph with "nfs_read" label on the $y$-axis shows the rate of NFS read calls per second. The last graph with the $y$-axis labeled "nfs_write" shows the rate of NFS write calls per second. These metrics represent the I/O activities of the application running on the system.

As we can see from the NFS data and based on the discussion in Section 5.2.1, the application starts with reading from input and configuration files. After this input activity, the initialization phase begins, which takes around 6 minutes. The initialization phase mostly involves mesh distribution and communication setup. Most of the calls to functions like MPI_Reduce and MPI_Scatter happen during this phase. After the initialization, the computation phase starts, which consists of five timesteps. 


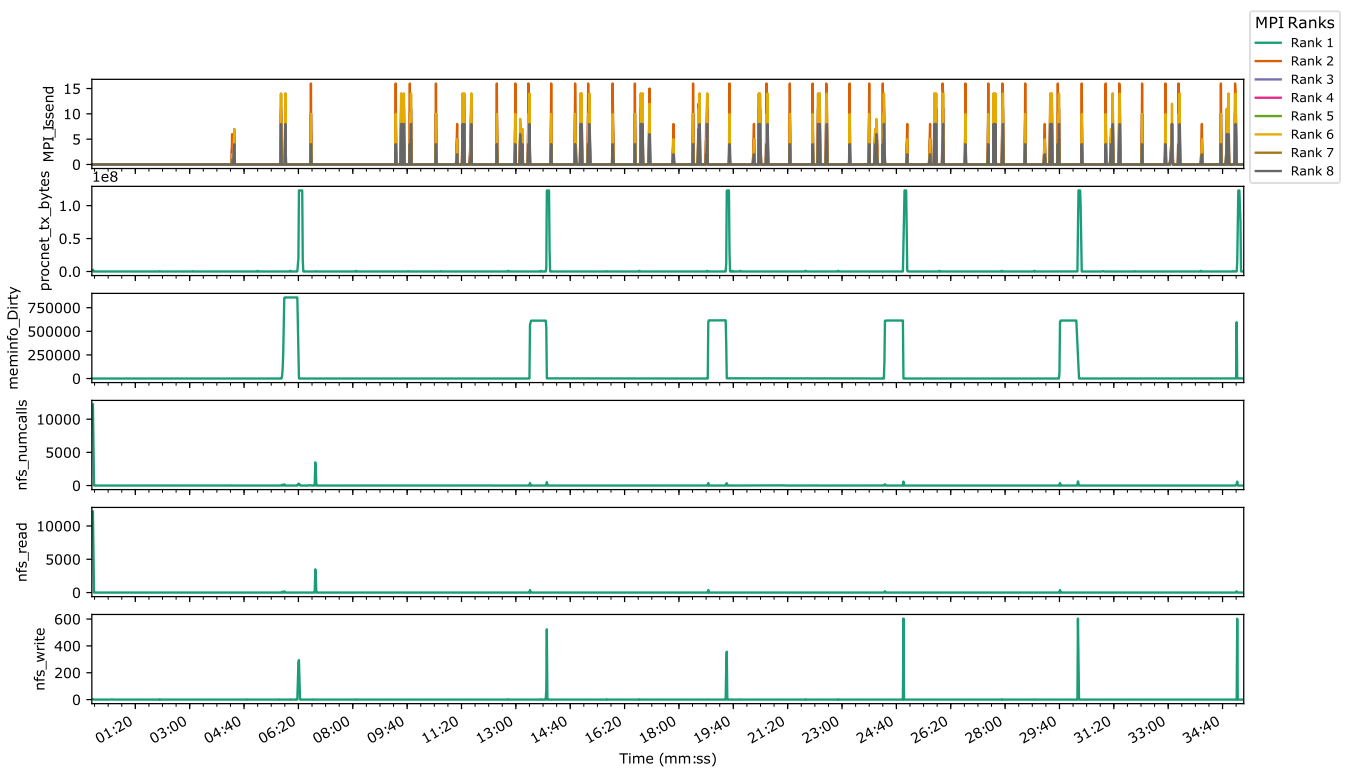

Fig. 7. Correlations between hardware and software data.

The first timestep in Nalu application begins with a sudden increase in the number of read calls to NFS. Here we can see how the application is interacting with the system using I/O operations, while it exhibits no signal of the MPI event. NFS reading is followed by a peak in Dirty metric of memory. This buffering in the NFS software stack is completed with a sudden increase in writing. This write event involves flushing the data, which is accumulated in memory and indicated by Dirty, to the disk. The next event is a sudden increase in the amount of data transmitted by the network interface, which is followed by a considerable number of calls to MPI_Issend. All of these events happened from the beginning of the timestep to the end of the MomentumEQS in the first nonlinear iteration.

From this point, we can see repeated computation periods interrupted by chunks of file output. The same pattern with a similar sequence of events repeats. Due to the $1 \mathrm{~Hz}$ relatively infrequent sampling rate, the profile peaks vary somewhat from one timestep to the next.

Using our tool-set, we can monitor the behavior of applications. Integrating with LDMS enables us to correlate application-level events with metrics that indicate system utilization. Streaming this performance data enables us to track application progress and detect unexpected behaviors as soon as they happen during the application runtime.

5.2.3 Anomaly Detection. One of the challenges in HPC system management is application performance variations that surprise the user. Resource contention in the presence of other processes is an anomaly example that can cause performance variations and potentially lost computing cycles [8]. The quick detection of performance variations is critical to mitigate the issues and improve resource utilization in a timely manner. Typical profiling tools fail to help detect such issues as they occur, because only post-run analysis methods are available. In this section, we show how our tool-set can help detect an abnormal behavior in Nalu.

We design an experiment to evaluate the capability of our tool-set to support the anomaly detection. In this experiment, we run Nalu in an environment where another application might be run simultaneously. We run this experiment on the Xeon system as specified in Table 1. Nalu 

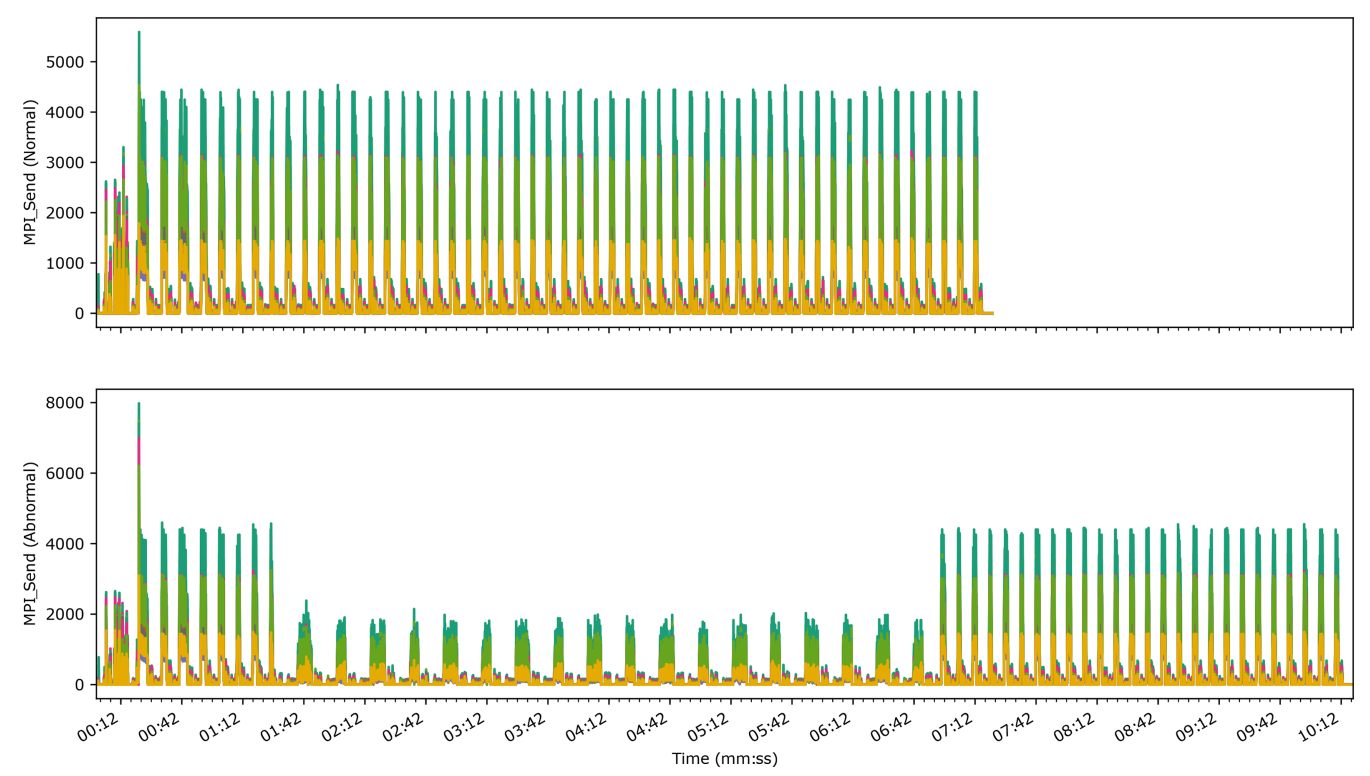

Fig. 8. Revealing abnormal behavior.

application takes the milestoneRun problem as the input and utilizes thirty MPI processes to run. We use our tool-set to investigate how the behavior of Nalu application changes in the presence of other processes.

In Figure 8, two graphs are plotted together. Both graphs share the same $x$-axis that represents the runtime in minutes. The $y$-axis on both graphs displays the rate of calls per 0.1 s to MPI_Send function. The first graph on the top represents the data collected from the normal run of Nalu application. The other graph on the bottom shows the data collected from the abnormal run where there is contention for system resources in the presence of other processes.

At the beginning of this experiment, we have Nalu and LDMS daemons as the only processes running on the system. After about 90s from the beginning of the execution of Nalu, other processes start on the system. The presence of other processes introduces a contention on system's resources. We can see this impact on the bottom graph in Figure 8 immediately after it occurs. The resource contentions cause a performance degradation. We can see that the duration of each application major iteration has increased by a factor of 2. At each phase, the rate of calls to MPI_Send function has decreased compared to the normal execution. The termination of the competing processes after 5 minutes restores normal operation. We can see that the rate of calls to MPI_Send function in the abnormal run has decreased by half compared to the regular execution.

Our tool-set can help detect an abnormal behavior in an application as soon as it occurs. The presented results demonstrate the benefits of our tool-set over the tracing tools in the anomaly detection. By streaming application-level events we can decrease the latency in the application analysis and issue mitigation. As in this case, determining the normal behavior for a set of metrics is application specific. Our tool-set can be coupled with automatic anomaly detection methods such as statistical data analysis approaches to automate the process.

5.2.4 Monitoring Multiple Applications. Our approach is designed to enable monitoring of multiple applications. The tool-set interacts with application processes and these processes can belong to different applications. In this section, we demonstrate how our tool-set can be used for streaming performance data collected from multiple applications running at the same time. 


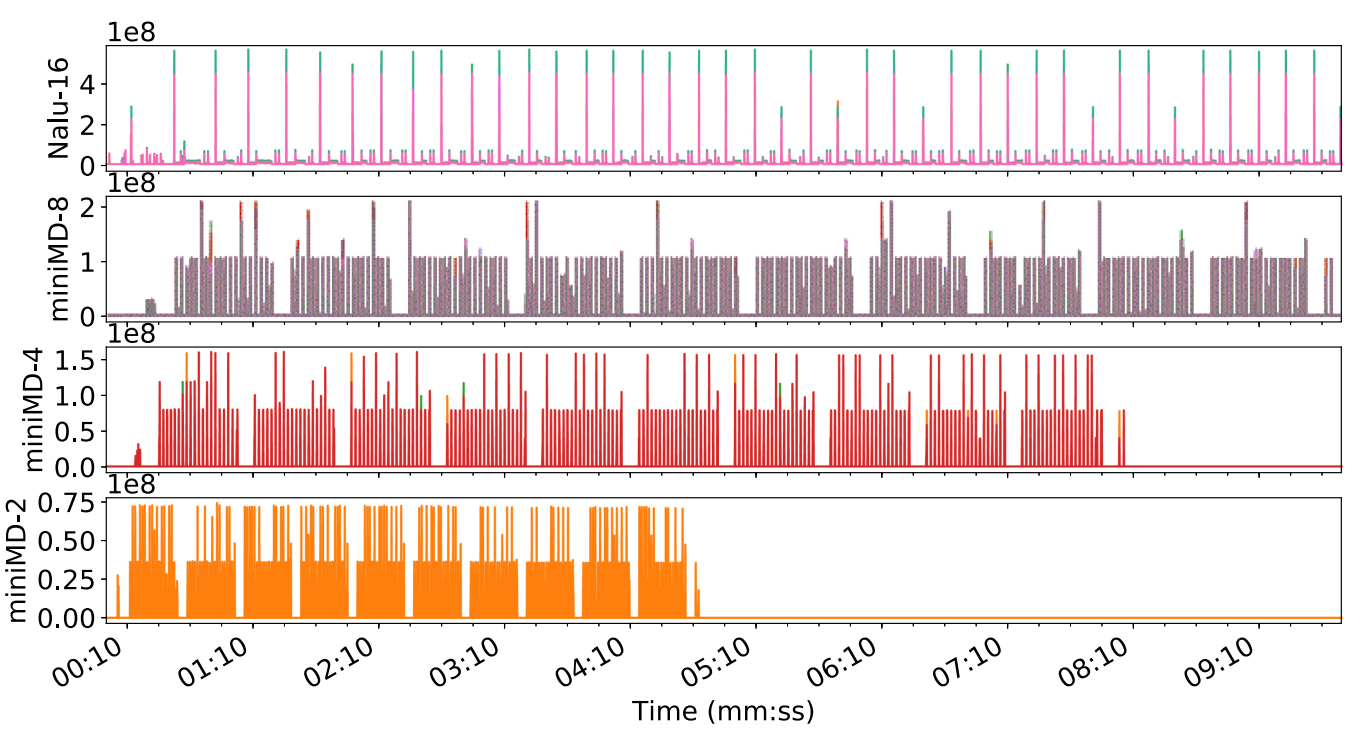

Fig. 9. Monitoring multiple applications. The $y$-axis represents the rate of messages sent using the MPI_Send function.

We design an experiment to evaluate the capability of our tool-set to monitor four running applications at the same time. In this experiment, we run one instance of Nalu and three instances of an MPI-based scientific mini-application, MiniMD. MiniMD is a Molecular Dynamics (MD) miniapplication in the Mantevo mini-application project [21]. It represents the computation and communications of the Lennard-Jones method used in the LAMMPS application [35]. We run this experiment on the Xeon system as specified in Table 1.

In our experiment, Nalu application takes the milestoneRun problem as the input and utilizes sixteen MPI processes to run. Each instance of the miniMD application is run using the default test with different problem sizes and number of MPI processes. The smallest instance is run using two MPI processes and 400,000 atoms. The next instance utilizes four MPI processes and has 13,500,000 atoms in the input configurations. The largest instance of the miniMD uses eight MPI processes to work on 32,000,000 atoms that specified in the input configurations.

In Figure 9, four graphs are plotted together. All graphs share the same $x$-axis that represents the runtime in minutes. The $y$-axis on all plots displays the rate of changes in the volume of messages that are communicated using the MPI_Send function. The first graph from the top with "Nalu16 " label on the $y$-axis represents the rate of messages for the Nalu application that is running using 16 processes. The second graph with "miniMD-8" label on the $y$-axis shows the message rate for the miniMD application when is run with 8 processes. The third graph with the $y$-axis labeled "miniMD-4" displays the rate of messages for the miniMD application when is run with 4 processes. The last graph with the $y$-axis labeled "miniMD-2" shows the message rate for another instance of the miniMD application that is run with two MPI processes.

As we can see in Figure 9, all applications start at the same time. Although the repetitive pattern can be seen in all of them, each application instance exhibits a different behavior depending on the problem and configuration. The miniMD-2 instance executes each iteration faster and send fewer messages compared to others, e.g., less than $50 \%$ of the miniMD- 8 messages. This application finishes its execution after around 5 minutes. The miniMD- 4 instance, which has longer iterations, finishes 3 minutes later. The long iteration and high number of messages of the miniMD- 8 instance indicates that it is solving a larger problem compared to other instances of miniMD. 
Another notable point is the difference between the behavior of applications in Figures 8 and 9 . In Section 5.2.3, an anomaly is injected to interfere with the computations of the main application and limit its available resources. Figure 8 shows how this interference leads to the abnormal behavior and the runtime slow-down. In our experiment in this section, we ensure the availability of computing cores to all applications. $50 \%$ of available cores have been used by the Nalu application. Three miniMD instances use 14 of the remaining $16 \mathrm{CPU}$ cores. As a result, we do not observe a significant slow-down in this experiment.

Our tool-set enables HPC users to monitor multiple applications. This is achieved by our approach's design that utilizes a specific channel for data collection from each application.

\subsection{Overhead Evaluation}

Our approach is intended for the deployment in a production environment on large-scale HPC systems. As a result, performance and scalability of the implementation become critical requirements. We investigate the overhead of using our tool-set to validate that our design meets the requirements. We design a set of experiments to investigate the overhead in different configurations. In our analysis, we perform the following impact assessments:

- Impact of using our tool-set compared to the base case with no monitoring.

- Impact of changing sampling frequency and data resolution.

- Impact of the presence of other LDMS samplers.

- Impact of using different strategies in process placements.

We run the experiments in the environments specified by Table 1 . To avoid hardware variability within result sets that may obscure small overhead costs, we run the related experiments in series on the same compute node.

We use two types of systems to run the experiments to understand the interaction of our tool-set with the operating system and applications on different HPC platforms. The first system includes compute nodes that are equipped with Intel Xeon Phi $72501.4 \mathrm{GHz}$ processors (68 cores), 16GB high-speed cache, and 96GB DDR4 memory. This architecture is used on some HPC platforms (e.g., Reference [13]) and may still be a representative of future architectures. CentOS 7.3 is running as the operating system. We build Nalu and its dependencies using Intel Compiler 17.0.1 enabled with the level three optimization. We refer to this system as KNL. The next system, which we refer to as Xeon, includes compute nodes that are equipped with Intel Xeon processors, one of the mainstream processors utilized in HPC systems. On this system, 32 Intel Xeon E5-2683 processors are running at $2.10 \mathrm{GH}$ with $128 \mathrm{~GB}$ memory. CentOS 7.1 is the operating system, and we build Nalu using Intel Compiler 17.0.1.

We run our tool-set alongside Nalu application to measure the overhead based on the wall time reported by Nalu's output log file. Nalu performs the simulation utilizing a computational mesh. In our experiments, we use an R2 mesh with 2725802 elements and 77MB file size. We choose a Nalu problem that is called milestoneRun from Nalu's regression test suite [15]. This problem has been used for Trinity acceptance tests [4]. We can use this problem to run Nalu with an arbitrary number of MPI processes.

We use $t$-test to verify the statistical significance of these results [23]. For each set of experiments, we compare two groups of results. One is the control group, which is defined according to the experiment and the type of impact assessment. The second group includes the results that are generated by changing the parameter related to the impact assessment experiment. For example, consider the experiment that we evaluate the impact of the process placement. In this experiment, we choose placement 1 as the baseline and the group of placement 1 results is the control group. The changing parameter in this experiment is the type of process placement. Any experiment 
Table 2. Process Placements Used in Experiments

\begin{tabular}{|c|c|c|c|c|}
\hline $\begin{array}{c}\text { Placement } \\
\text { Version }\end{array}$ & $\begin{array}{c}\text { \# MPI Ranks } \\
\text { KNL } \mid \text { Xeon }\end{array}$ & $\begin{array}{c}\text { Pinned/ } \\
\text { Unpinned }\end{array}$ & $\begin{array}{c}\text { LDMS Sampler Daemon } \\
\text { Processor Place }\end{array}$ & $\begin{array}{c}\text { LDMS Aggregator } \\
\text { Daemon Processor Place }\end{array}$ \\
\hline 1 & $272 \mid 32$ & Unpinned & Free & Free \\
\hline 2 & $271 \mid 31$ & Unpinned & Free & Free \\
\hline 3 & $270 \mid 30$ & Unpinned & Free & Free \\
\hline 4 & $272 \mid 32$ & Pinned & First & First \\
\hline 5 & $271 \mid 31$ & Pinned & First & First \\
\hline 6 & $270 \mid 30$ & Pinned & First & Last \\
\hline 7 & $272 \mid$ N/A & Pinned & Last & Last \\
\hline 8 & $271 \mid$ N/A & Pinned & Last & Second to last \\
\hline 9 & $270 \mid$ N/A & Pinned & Last & st \\
\hline
\end{tabular}

Numbers on the second column represent the number of MPI ranks we use to run Nalu. The third column states whether the processes were pinned or not. The fourth and fifth columns show core number that we use to run the sampler and aggregator daemons with pinning.

with a new process placement generates a new set of results. Every set of results form the second group for $t$-test to compare with the control group. This test demonstrates statistically significant results of our experiments with the $p$-value less than the chosen threshold of 0.01 .

5.3.1 Process Placement Strategies. We used several process placement strategies to measure Nalu's performance in different situations. Table 2 presents these strategies. In the first three placements, all MPI processes and LDMS daemons are unpinned. In the first placement, we do not reserve any processor for LDMS daemons, and the application utilizes all available processors, i.e., 272 on KNL and 32 on Xeon. We keep one and two processors idle in placements 2 and 3, respectively, and the MPI application uses the rest of the processors. In placements 4-6, we pin all MPI processes and LDMS daemons. The MPI application utilizes all processors in placement 4, and both LDMS sampler and aggregator are pinned to the first processor, which results in it being used by three processes. In placement 5 , we do not pin any MPI process to the first processor and use it for both sampler and aggregator. In placement 6 , we dedicate the first and second processors to the sampler and aggregator and pin 270 MPI ranks to the next 270 processors on KNL or 30 MPI ranks to the next 30 processors on Xeon. In placements $7-9$, we apply strategies similar to placements 4-6 with a difference that the last two, instead of the first two, processors are used for LDMS sampler and aggregator.

Previous generations of Intel Xeon Phi processors were known for reserving the first core for the operating system [27]. In our experiments, we take two approaches based on the reserving first or last cores for OS activities and LDMS daemons (if available) to investigate this impact. Since this is not the case for Xeon processors, we do not run experiments in placements 7-9 for Xeon.

Figure 10 exhibits Nalu's performance regarding runtime using different process placement strategies on both KNL (Figure 10(a)) and Xeon (Figure 10(b)). The vertical axis on both plots shows runtime in milliseconds and the horizontal axis presents different placement strategies as described in Table 2 . The rotated plot outside of the box shows the probability density of the data at different values [22]. In this figure, we exclude all data from the cases where we run LDMS.

In KNL experiments, Nalu demonstrates the best runtime when using the placement 6, where we keep the first two processors idle and use the rest of processors (270) as MPI ranks to run the application. Since we did not run LDMS in these experiments, the first two processors are free to be used by the system processes. The availability of these processors reduces the OS noise and its impact on the application performance and runtime variability. OS activity has been known as one 

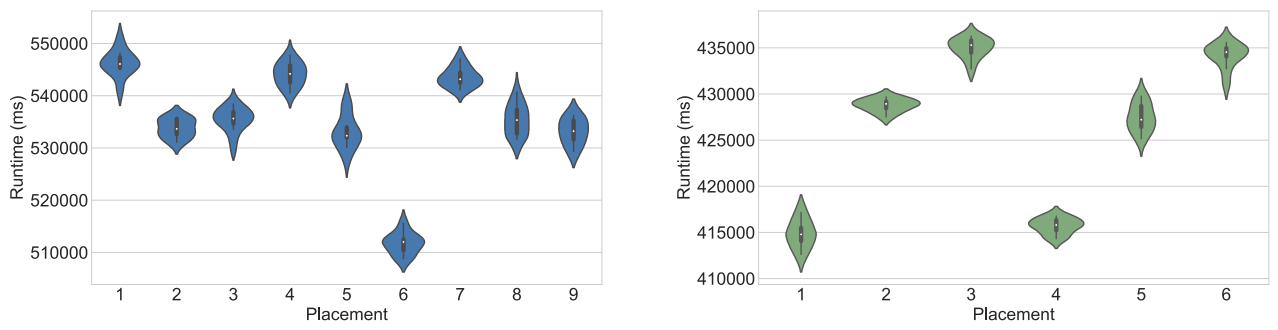

(a) Run-time of Nalu application on the KNL cluster. (b) Run-time of Nalu application on the Xeon cluster.

Fig. 10. Runtime of Nalu application with different process placement strategies when running on the KNL cluster (Figure 10(a)) and Xeon cluster (Figure 10(b)) without any LDMS daemons running. The runtime plot for each placement features a kernel density estimation of the underlying distribution of runtime derived from 10 samples. Table 2 provides a brief overview of different placements.

of the primary causes of the variability [6] and some methods exist to address this issue on Intel Xeon Phi processors [11].

We observe the highest runtime in placements 1,4 , and 7 . The overhead introduced by using these strategies are in the range of $6.5 \%$ on average. In all of these placements, we use the maximum available processors to run Nalu application, and system noise and necessary OS activities do not have any choices other than interrupting tasks being carried out by the MPI application. Choosing any of placements $2,3,5,8$, and 9 roughly adds $4.5 \%$ overhead on average to the application.

In Xeon experiments, Nalu exhibits the best runtime when we use all 32 available processors as MPI ranks. In general, using more processors on the Xeon machine allows Nalu to leverage the maximum parallelism. Pinning MPI processes does not seem to provide any benefits on Xeon. Placements 3 and 6 that keep two processors idle show the highest runtime with an overhead of $4.7 \%$ on average. Choosing any of placements 2 , and 5 roughly adds $3.2 \%$ overhead on average to the application's runtime. Placement 4 performs slightly slower by adding $0.2 \%$ overhead to the runtime of the placement 1 that differs only in pinning the MPI processes.

Given the best results we get from the placement 6 on KNL, and placement 1 on Xeon, we use these placements for the impact analysis of using our tool-set for monitoring Nalu.

5.3.2 Sampling Frequency Impact. In this section, we study how using our tool-set affects the performance of Nalu. We run experiments in two cases: regular Nalu run with no LDMS daemons running and a Nalu run where LDMS MPI sampler is running at the same time and data are collected from the application. We run the LDMS MPI sampler with four different frequencies: $0.1 \mathrm{~Hz}$, $1 \mathrm{~Hz}, 2 \mathrm{~Hz}$, and $10 \mathrm{~Hz}$.

Figure 11 depicts Nalu's performance regarding runtime on both KNL (Figure 11(a)) and Xeon (Figure 11(b)) when LDMS daemons are not running and the cases where the application is monitored with four different sampling frequencies. The vertical axis on both plots shows runtime in milliseconds and the horizontal axis displays different cases based on the sampling approach. For $\mathrm{KNL}$, we run these experiments using the placement 6 where the first two processors are used by LDMS daemons and the MPI application utilizes the last 270 processors. We use the placement 1 for running experiments on Xeon where we use all 32 available processors and do not pin any processes.

In KNL experiments, the overhead introduced by using our tool-set on average is within the range of $0.78 \%$ in different sampling frequencies. Running the sampler with the high frequency of $10 \mathrm{~Hz}$, on average, yields a surprisingly acceptable runtime. However, the runtime variability is 


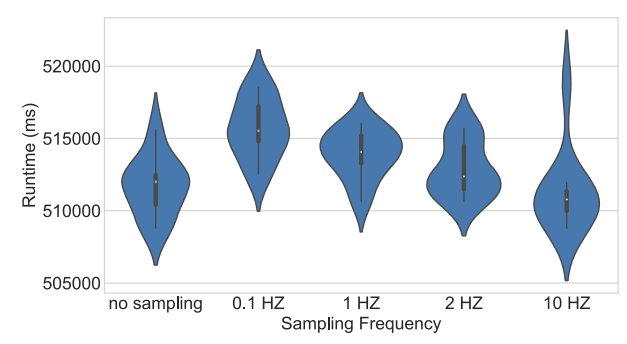

(a) Run-time of Nalu application on the KNL cluster (b) using placement 6 .

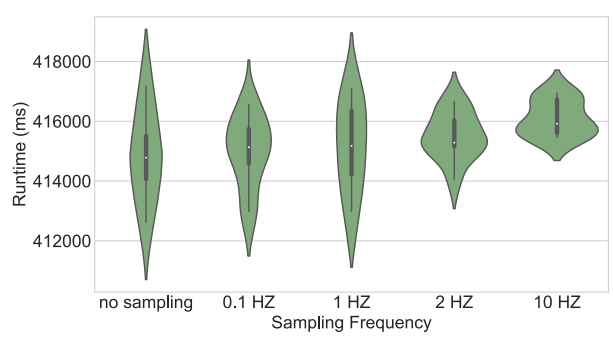

(b) Run-time of Nalu application on the Xeon cluster using placement 1 .

Fig. 11. Runtime of Nalu in the cases of running without monitoring and when LDMS daemons are running on the system with different MPI sampling frequencies. The runtime plot for each sampling case features a kernel density estimation of the underlying distribution of runtime derived from 10 samples.

Table 3. Sampling Versions

\begin{tabular}{|c|c|c|}
\hline $\begin{array}{c}\text { Sampling } \\
\text { Version }\end{array}$ & Running Samplers & $\begin{array}{c}\text { \# Collected Metrics } \\
\text { per Second KNL | Xeon }\end{array}$ \\
\hline 1 & MPI Sampler, vmstat, meminfo, procnetdev, procnfs, procstat & $5,912 \mid 903$ \\
\hline 2 & MPI Sampler & $2,700 \mid 320$ \\
\hline 3 & vmstat, meminfo, procnetdev, procnfs, procstat & $3,212 \mid 583$ \\
\hline 4 & None & $0 \mid 0$ \\
\hline
\end{tabular}

high in this case. Running the sampler at $0.1 \mathrm{~Hz}$ does not gain performance and, in some cases, it slightly slows down the application more than higher frequencies. Furthermore, we have lower data accuracy in this case.

Running the sampler at $1 \mathrm{~Hz}$ and $2 \mathrm{~Hz}$ leads to both reasonable performance and data accuracy. The runtime overhead introduced by both is less than $0.5 \%$. We can see some differences in the data distribution between these two frequencies. The $2 \mathrm{~Hz}$ case shows another small peak in higher runtime values in addition to the main peak that we have in all cases. However, the $1 \mathrm{~Hz}$ case demonstrates a distribution closer to normal with one peak.

In Xeon experiments, using our tool-set with the high frequency of $10 \mathrm{~Hz}$ and $2 \mathrm{~Hz}$, respectively adds $0.3 \%$ and $0.16 \%$ overhead on average to the runtime. The runtime overhead of running the sampler at $1 \mathrm{~Hz}$ and $0.1 \mathrm{~Hz}$ are both less than $0.1 \%$. Other than the lower overhead, the higher accuracy in data collected by the sampler at $1 \mathrm{~Hz}$ and its distribution distinguish this case from other cases.

This outcome suggests that we choose to run the samplers at $1 \mathrm{~Hz}$ for our next study to get a fair result.

5.3.3 Impact of the Presence of Other Samplers. Collecting data from software and different hardware components at the same time helps gain a comprehensive understanding of the application behavior.

In this section, we study the impact of running the MPI sampler alongside other samplers on the runtime of Nalu application.

We run the experiments with four different versions of sampling for this study. Table 3 presents the information about each version. In version 1, we run all of the samplers including the MPI sampler. In version 2, we only run the MPI sampler. All samplers but the MPI sampler are evaluated in the version 3 . We also have a regular application run in version 4 , where no sampler is running. Other samplers provide statistics related to the virtual memory, RAM and 

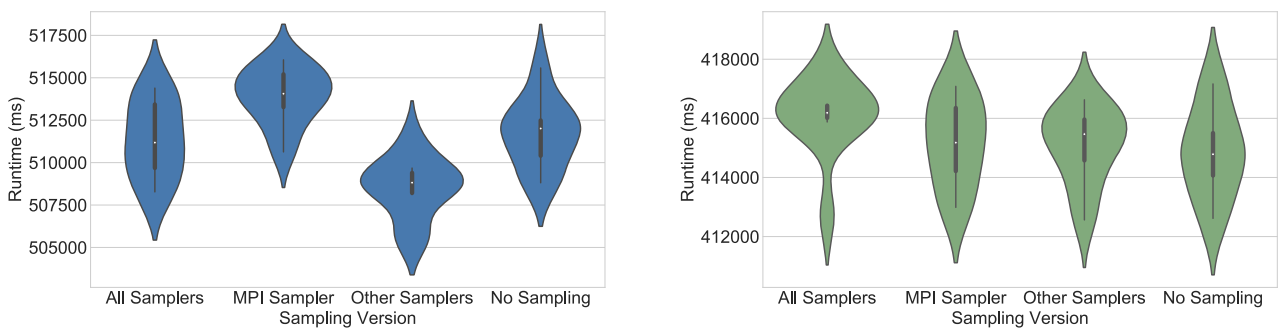

(a) Run-time of Nalu application on the KNL cluster. (b) Run-time of Nalu application on the Xeon cluster.

Fig. 12. Runtime of Nalu in the case of running without monitoring and of many LDMS samplers running on the system. The runtime plot for each sampling case features a kernel density estimation of the underlying distribution of runtime derived from 10 samples. Table 3 provides a brief overview of different sampling versions. All of the samplers collect data at $1 \mathrm{~Hz}$.

CPU utilization, networking, and file systems. The last column of this table indicates the number of metrics that are collected and exposed by samplers at each second during the execution.

Based on the findings from previous sections, for KNL experiments, we use placement 6, where the first two processors are reserved to be used by LDMS daemons if present. For Xeon experiments, we use placement 1 , where we use all available processors with no pinning involved. When we run LDMS samplers, i.e., versions $1-3$, we set the sampling frequency to $1 \mathrm{~Hz}$.

Figure 12 displays Nalu's performance regarding runtime on both KNL (Figure 12(a)) and Xeon (Figure 12(b)) when LDMS daemons are not running and the cases where the application is monitored with many LDMS samplers. The vertical axis on both plots shows runtime in milliseconds and the horizontal axis represents different sampling versions as described in Table 3.

In KNL experiments, the overhead introduced by the samplers in different cases is within the range of $0.5 \%$ of the base case, i.e., no sampling. We observe that running all samplers, where we roughly collect 6,000 metrics every second, causes the runtime to be more scattered across the data range. The non-MPI samplers collect more than 3,200 metrics from different data sources every second. Most of these hardware-level data are provided in /proc file system. However, MPI sampler uses the shared memory index to expose the 2,700 metrics collected by the MPI profiler. This seems to impact the application's runtime more than other hardware-level samplers. The overhead is still within the range of $0.5 \%$ of the original application run. A group of HPC users at SNL provided bounds on acceptable overhead to be less than $1 \%$ slowdown [2].

In Xeon experiments, the overhead introduced by the samplers in different cases, on average, is within the range of $0.3 \%$ of the base case, i.e., no sampling. When LDMS collects 900 metrics per second using all samplers, we observe the highest overhead of $0.29 \%$. Using LDMS MPI sampler alone increases the runtime by less than $0.1 \%$.

Impact analysis results show that the overhead introduced by our tool-set is within the acceptable overhead range. This makes our tool-set a suitable choice to deploy in a production environment for continuous monitoring.

Experimental evaluation results demonstrate that our low overhead tool-set helps understand the behavior of applications by streaming software-level performance data. Our approach enables runtime operational analysis by exploring the correlations between hardware and software-level data.

\section{CONCLUSION AND FUTURE WORK}

We have developed and demonstrated a hybrid approach to HPC application monitoring that supports performance analysis in production conditions. This approach takes advantage of the low 
overhead of shared memory and LDMS to provide insights into the application's behavior using profiling during the execution. By taking this approach, we avoid the overhead of heavy tracing methods. We have implemented a tool-set to evaluate its impact. While we demonstrate our approach using MPI applications, it does not require a specific programming paradigm and the design is generally applicable. We integrated our tool-set with the LDMS framework to create a scalable hybrid tool that streams application performance data using profiling. By taking advantage of the shared memory approach, components within our tool-set can communicate efficiently. We have resolved challenges of using this method and provided mechanisms for fault management.

We have evaluated our approach using test cases of the open source HPC code, Nalu. We have shown how our tool-set provides insights that can be used in combination with other data sources to reveal application phases during the execution without access to application source code. By integrating with LDMS, we have run experiments that stream several types of data using hardware samplers in addition to the software-level metrics collected by our tool-set during the execution. We provided examples that show how this tool-set helps explore the correlations between different events happening during the execution. Also, we demonstrated how our tool-set helps us to detect abnormal behavior in an application during the execution.

Overhead results show a slight increase in runtime, which is within the acceptable range. This feature makes our approach a suitable choice for continuous performance monitoring in a production environment. Integrating this approach with LDMS enables streaming performance data to storage efficiently and supports runtime analysis and feedback to the application.

Future work includes deriving insights from the data obtained by our tool-set and leveraging that for the automatic identification of events during the application execution in the production environment. We seek to discover possible issues such as application stalls and enable mechanisms to avoid undesirable events. Another possible direction is to devise and implement similar methodologies for monitoring other types of applications such as multi-threaded programs, and parallel applications enabled with non-blocking or transactional data structures.

\section{ACKNOWLEDGMENTS}

The authors acknowledge the University of Central Florida Advanced Research Computing Center and Fujitsu (in partnership with Intel) for providing computational resources and support that have contributed to the results reported herein.

\section{REFERENCES}

[1] Laksono Adhianto, Sinchan Banerjee, Mike Fagan, Mark Krentel, Gabriel Marin, John Mellor-Crummey, and Nathan R. Tallent. 2010. HPCToolkit: Tools for performance analysis of optimized parallel programs. Concurr. Comput.: Pract. Exp. 22, 6 (2010), 685-701.

[2] Anthony Agelastos, Benjamin Allan, Jim Brandt, Paul Cassella, Jeremy Enos, Joshi Fullop, Ann Gentile, Steve Monk, Nichamon Naksinehaboon, Jeff Ogden, et al. 2014. The lightweight distributed metric service: A scalable infrastructure for continuous monitoring of large scale computing systems and applications. In Proceedings of the International Conference for High Performance Computing, Networking, Storage and Analysis. IEEE, Los Alamitos, CA, 154-165.

[3] Anthony Agelastos, Benjamin Allan, Jim Brandt, Ann Gentile, Sophia Lefantzi, Steve Monk, Jeff Ogden, Mahesh Rajan, and Joel Stevenson. 2016. Continuous whole-system monitoring toward rapid understanding of production HPC applications and systems. Parallel Comput. 58 (2016), 90-106.

[4] A. M. Agelastos, M. Rajan, N. Wichmann, R. Baker, S. Domino, E. W. Draeger, S. Anderson, J. Balma, S. Behling, M. Berry, et al. 2017. Performance on Trinity phase 2 (a Cray XC40 utilizing Intel Xeon Phi processors) with acceptance applications and benchmarks. Cray User Group CUG, May (2017). https://cug.org/proceedings/cug2017_proceedings/ includes/files/pap138s2-file1.pdf.

[5] Anthony Michael Agelastos and Paul T. Lin. 2013. Simulation information regarding sandia national laboratories' Trinity capability improvement metric. Sandia National Laboratories, Albuquerque, New Mexico. https://prod-ng. sandia.gov/techlib-noauth/access-control.cgi/2013/138748.pdf. 
[6] Pete Beckman, Kamil Iskra, Kazutomo Yoshii, Susan Coghlan, and Aroon Nataraj. 2008. Benchmarking the effects of operating system interference on extreme-scale parallel machines. Cluster Comput. 11, 1 (2008), 3-16.

[7] Shajulin Benedict, Ventsislav Petkov, and Michael Gerndt. 2010. Periscope: An online-based distributed performance analysis tool. In Tools for High Performance Computing 2009. Springer, Berlin, 1-16.

[8] Abhinav Bhatele, Kathryn Mohror, Steven H. Langer, and Katherine E. Isaacs. 2013. There goes the neighborhood: Performance degradation due to nearby jobs. In Proceedings of the International Conference on High Performance Computing, Networking, Storage and Analysis. ACM, New York, NY, 41.

[9] J. Brandt, A. Gentile, M. Showerman, Jeremy Enos, Joshi Fullop, and G Bauer. 2016. Large-scale persistent numerical data source monitoring system experiences. In Proceedings of the 2016 IEEE International Parallel and Distributed Processing Symposium Workshops. IEEE, Los Alamitos, CA, 1711-1720.

[10] Shirley Browne, Jack Dongarra, Nathan Garner, George Ho, and Philip Mucci. 2000. A portable programming interface for performance evaluation on modern processors. Int. f. High Perf. Comput. Appl. 14, 3 (2000), 189-204.

[11] Sudheer Chunduri, Kevin Harms, Scott Parker, Vitali Morozov, Samuel Oshin, Naveen Cherukuri, and Kalyan Kumaran. 2017. Run-to-run variability on Xeon Phi based cray XC systems. In Proceedings of the International Conference for High Performance Computing, Networking, Storage and Analysis. ACM, 52.

[12] Adam DeConinck, A. Bonnie, K. Kelly, S. Sanchez, C. Martin, M. Mason, James M. Brandt, Ann C. Gentile, Benjamin A. Allan, Anthony Michael Agelastos, and others. Design and Implementation of a Scalable Monitoring System for Trinity. Sandia National Lab.(SNL-NM), Albuquerque, NM (United States). https://cug.org/proceedings/ cug2016_proceedings/includes/files/pap126s2-file2.pdf.

[13] Adam DeConinck, Hai Ah Nam, David Morton, Amanda Bonnie, Cory Lueninghoener, James M. Brandt, Ann C. Gentile, Kevin Pedretti, Anthony Michael Agelastos, Courtenay T. Vaughan and others. 2017. Runtime collection and analysis of system metrics for production monitoring of Trinity Phase II. Sandia National Lab.(SNL-NM), Albuquerque, NM (United States). https://cug.org/proceedings/cug2017_proceedings/includes/files/pap126s2-file1.pdf.

[14] Jason Dixon. 2017. Monitoring with Graphite: Tracking Dynamic Host and Application Metrics at Scale. O'Reilly Media, Inc.

[15] S. Domino. 2018. Nalu's milestoneRun test at master. Retrieved from https://github.com/NaluCFD/Nalu/tree/master/ reg_tests/test_files/milestoneRun.

[16] S. Domino. 2018. Sierra Low Mach Module: Nalu Theory Manual 1.0. Retrieved from https://github.com/NaluCFD/ NaluDoc.

[17] Steven Feldman, Deli Zhang, Damian Dechev, and James Brandt. 2015. Extending LDMS to enable performance monitoring in multi-core applications. In Proceedings of the 2015 IEEE International Conference on Cluster Computing. IEEE, $717-720$.

[18] Todd Gamblin, Martin Schulz, Bronis R. de Supinski, Felix Wolf, Brian J. N. Wylie, et al. 2011. Reconciling sampling and direct instrumentation for unintrusive call-path profiling of MPI programs. In Proceedings of the IEEE International Parallel \& Distributed Processing Symposium (IPDPS'11). IEEE, 640-651.

[19] Markus Geimer, Felix Wolf, Brian J. N. Wylie, Erika Ábrahám, Daniel Becker, and Bernd Mohr. 2010. The Scalasca performance toolset architecture. Concurr. Comput.: Pract. Exper. 22, 6 (2010), 702-719.

[20] Susan L. Graham, Peter B. Kessler, and Marshall K. Mckusick. 1982. Gprof: A call graph execution profiler. In ACM Sigplan Notices, Vol. 17. ACM, 120-126.

[21] Michael A. Heroux, Douglas W. Doerfler, Paul S. Crozier, James M. Willenbring, H. Carter Edwards, Alan Williams, Mahesh Rajan, Eric R. Keiter, Heidi K. Thornquist, and Robert W. Numrich. 2009. Improving performance via mini-applications. Sandia National Laboratories, Tech. Rep. SAND2009-5574 3. http://www.sandia.gov/ maherou/ docs/MantevoOverview.pdf.

[22] Jerry L. Hintze and Ray D. Nelson. 1998. Violin plots: A box plot-density trace synergism. Am. Stat. 52, 2 (1998), 181-184.

[23] Torsten Hoefler and Roberto Belli. 2015. Scientific benchmarking of parallel computing systems. In Proceedings of the International Conference for High Performance Computing, Networking, Storage and Analysis.

[24] Thomas Ilsche, Joseph Schuchart, Robert Schöne, and Daniel Hackenberg. 2015. Combining instrumentation and sampling for trace-based application performance analysis. In Tools for High Performance Computing 2014. Springer, Berlin, 123-136.

[25] Ramin Izadpanah, Steven Feldman, and Damian Dechev. 2016. A methodology for performance analysis of nonblocking algorithms using hardware and software metrics. In Proceedings of the IEEE 19th International Symposium on Real-Time Distributed Computing (ISORC'16). IEEE, 43-52.

[26] Ramin Izadpanah, Nichamon Naksinehaboon, Jim Brandt, Ann Gentile, and Damian Dechev. 2018. Integrating lowlatency analysis into HPC system monitoring. In Proceedings of the 47th International Conference on Parallel Processing. ACM, 5 . 
[27] Jim Jeffers and James Reinders. 2015. High Performance Parallelism Pearls Volume Two: Multicore and Many-core Programming Approaches. Morgan Kaufmann.

[28] I. S. Kadochnikov, N. A. Balashov, A. V. Baranov, I. S. Pelevanyuk, N. A. Kutovskiy, V. V. Korenkov, and A. V. Nechaevskiy. 2016. Evaluation of monitoring systems for metric collection in intelligent cloud scheduling. In Proceedings of the CEUR Workshop, Vol. 1787. 279-283.

[29] Edward Karrels and Ewing Lusk. 1994. Performance analysis of MPI programs. In Proceedings of the Workshop on Environments and Tools for Parallel Scientific Computing. 195-200.

[30] Michael Kerrisk. 2010. The Linux Programming Interface. No Starch Press.

[31] Andreas Knüpfer, Holger Brunst, Jens Doleschal, Matthias Jurenz, Matthias Lieber, Holger Mickler, Matthias S. Müller, and Wolfgang E. Nagel. 2008. The vampir performance analysis tool-set. In Tools for High Performance Computing. Springer, Berlin, 139-155.

[32] Andreas Knüpfer, Christian Rössel, Dieter an Mey, Scott Biersdorff, Kai Diethelm, Dominic Eschweiler, Markus Geimer, Michael Gerndt, Daniel Lorenz, Allen Malony, et al. 2012. Score-P: A joint performance measurement runtime infrastructure for Periscope, Scalasca, TAU, and Vampir. In Tools for High Performance Computing 2011. Springer, Berlin, 79-91.

[33] Los Alamos National Laboratory. 2018. Trinity. Retrieved from https://www.lanl.gov/projects/trinity.

[34] Aniruddha Marathe, Hormozd Gahvari, Jae-Seung Yeom, and Abhinav Bhatele. 2016. LibPowerMon: A lightweight profiling framework to profile program context and system-level metrics. In Proceedings of the 2016 IEEE International Parallel and Distributed Processing Symposium Workshops. IEEE, Los Alamitos, CA, 1132-1141.

[35] S. S. Antman J. E. Marsden, L. Sirovich S. Wiggins, L. Glass, R. V. Kohn, and S. S. Sastry. 1993. Interdisciplinary Applied Mathematics. Vol. 3. Springer, Berlin.

[36] Matthew L. Massie, Brent N. Chun, and David E. Culler. 2004. The Ganglia distributed monitoring system: Design, implementation, and experience. Parallel Comput. 30, 7 (2004), 817-840.

[37] Bernd Mohr, Darryl Brown, and Allen Malony. 1994. TAU: A portable parallel program analysis environment for $\mathrm{C}++$. In Proceedings of the Parallel Processing: CONPAR 94-VAPP VI. Springer, 29-40.

[38] National Center for Supercomputing Applications. 2018. Blue Waters. Retrieved from https://bluewaters.ncsa.illinois. edu.

[39] Prometheus authors. 2018. Prometheus-Monitoring System and Time Series Database. Retrieved from https:// prometheus.io.

[40] J. Reams. 2012. Extensible monitoring with Nagios and messaging middleware. In Strategies, Tools, and Techniques: Proceedings of the 26th Large Installation System Administration Conference (LISA'12). 153-162.

[41] Thomas Röhl, Jan Eitzinger, Georg Hager, and Gerhard Wellein. 2017. LIKWID monitoring stack: A flexible framework enabling job specific performance monitoring for the masses. In Proceedings of the IEEE International Conference on Cluster Computing (CLUSTER'17). IEEE, 781-784.

[42] Syed Mansoor Sarwar and Robert M. Koretsky. 2016. UNIX: The Textbook. CRC Press, Boca Raton, FL.

[43] Martin Schulz, Jim Galarowicz, Don Maghrak, William Hachfeld, David Montoya, and Scott Cranford. 2008. Open SpeedShop: An open source infrastructure for parallel performance analysis. Sci. Program. 16, 2-3 (2008), 105-121.

[44] Harald Servat, Germán Llort, Judit Giménez, and Jesús Labarta. 2009. Detailed performance analysis using coarse grain sampling. In Proceedings of the European Conference on Parallel Processing. Springer, 185-198.

[45] David Skinner. 2005. Performance monitoring of parallel scientific applications. Ernest Orlando Lawrence Berkeley National Laboratory, Berkeley, CA (US). https://www.osti.gov/servlets/purl/881368.

[46] Sudharshan S. Vazhkudai, Ross Miller, Devesh Tiwari, Christopher Zimmer, Feiyi Wang, Sarp Oral, Raghul Gunasekaran, and Deryl Steinert. 2017. GUIDE: A scalable information directory service to collect, federate, and analyze logs for operational insights into a leadership HPC facility. In Proceedings of the International Conference for High Performance Computing, Networking, Storage and Analysis. ACM, 45.

[47] Jeffrey Vetter and Chris Chambreau. 2005. mpiP: Lightweight, Scalable MPIProfiling. http://mpip.sourceforge.net/

[48] Jonathan Stuart Ward and Adam Barker. 2014. Observing the clouds: A survey and taxonomy of cloud monitoring. f. Cloud Comput. 3, 1 (2014), 24.

Received March 2018; revised January 2019; accepted February 2019 